\title{
Weak evidence of caries reduction using xylitol-based lozenges in two of three selected studies
}

\section{Abstracted from}

Antonio AG, Pierro VS, Maia LC.

Caries preventive effects of xylitol-based candies and lozenges: a systematic review. J Public Health Dent 2011 71: 117-124.

Address for correspondence: Maia, LC Department of Pediatric Dentistry and Orthodontics,

Federal University of Rio de Janeiro, Caixa Postal 68066, Cidade Universitaria- CCS. 21941971, Rio de Janeiro, Brazil.

\section{Question: Does caries progress in subjects who consume xylitol based candies or lozenges for at least one year?}

Data sources The Medline, PubMed, ISI Web of Science, LILACS, and Cochrane Library databases and articles from the references of identified articles.

Study selection Controlled clinical trials and randomised controlled clinical trials (RCTs) of at least one year investigating the link between consuming xylitol-based lozenges; and caries increment; when compared with a control, in any population group.

Data extraction and synthesis Two examiners independently evaluated the titles and abstracts of all clinical trials identified in both the electronic and hand searches. Methodological quality was assessed using methods adapted from Chambrone et al. ${ }^{1}$ The main outcome was change in caries as measured by $\mathrm{WHO}$ criteria and the measure of treatment effect was Prevented Fraction(PF).

Results One hundred and twenty-seven non duplicate references were identified. From these, three studies were evaluated. Of the three studies two found a lower caries increment in the xylitol-based groups. The third found no difference in caries prevention between control and intervention.

Conclusions There is a need for well-designed randomised clinical studies with adequate control groups and high compliance by the subjects.

\section{Commentary}

There is a growing body of research on Xylitol use in dentistry as shown by the fact that a simple search of PubMed using just the terms 'xylitol and dental' reveals more than 560 studies. Traditionally researchers believed that the effectiveness of xylitol was related to its use as a sugar substitute. ${ }^{2}$ Others believe that chewing-gum sweetened with xylitol reduces caries solely through the chewing-gum's ability to cause salivary stimulation. ${ }^{3}$ The European Food and Safety Authority ${ }^{4}$ (EFSA) stated that despite poor allocation concealment and poor controlling for confounders in the available research, that there was a cause and effect relationship between the use of $100 \%$ xylitol sweetened chewing-gum and the reduction of tooth decay in children. The evidence for specific antibacterial properties of xylitol, primarily through its ability to interfere with the metabolism of Streptococcus mutans, is increasing. ${ }^{5-8}$ In practice xylitol could be efficacious regardless of the carrier, a number of which have been trialled, these include lozenges, candy (eg gummy bears $)^{9}$ and syrup. ${ }^{10}$

Antonio provides a comprehensive review of the current research around xylitol based candies and lozenges. The review question, while not phrased in an exact $\mathrm{PICO}^{11}$ format, is well focused. A comprehensive abstract is provided with objectives, methods, results and conclusions. PRISMA (www.prisma-statement.org/statement.htm) provides helpful recommendations as to what could be included in an abstract. In this instance a description of the importance of the systematic review and listing the data sources used to search for papers within the abstract would have been helpful. In the body of the report all information sources and strategies are described, with dates enabling the search to be repeated. The process of selecting studies is well described, and a flow diagram is included with detailed explanations of included or excluded papers, with additional information available online.

As the authors note, this review is limited in the fact that grey literature was not reviewed. There is now evidence that trials which are not formally published have results that differ systematically from published articles, usually showing less benefit of treatment. ${ }^{12}$ Consequently, time spent identifying grey literature (eg from clinical trials databases, or European literature or System for Information on Grey Literature in Europe [SIGLE] www.opengrey.eu ) can be valuable especially in an area of research where papers are limited.

Antonio provides a number of informative tabulated descriptions of the three papers reviewed. The inclusion of dmfs (decayed, missing, and filled surfaces) scores within these tables enables the reader to determine for each paper the similarity of the patient group 


\section{CARIES}

to their own population. Although these tables could have been improved by describing the control group and follow up period of study for each paper.

All papers had the summary measure of $\mathrm{PF}$, standard error (SE) along with 95\% confidence interval (CI). The measure of standard deviation is advantageous as it enables easy calculations of data spread, and can demonstrate clear relationships between the mean and standard deviations.

As the authors indicate, the three studies are quite different due to the daily dosage of xylitol used, discrepancy between the caries risk status and baseline caries profile, and the type of surface that was evaluated. ${ }^{13}$ Such heterogeneity means it can be difficult to meaningfully use a forest plot to graphically illustrate the results from the three studies. Similarly no meta-analysis, sensitivity analysis or subgroup analysis could be logically performed due to both the size and the heterogeneity of the included studies.

The quality of the included papers was assessed as unclear due to poor methods of randomisation, ${ }^{14,15}$ undetermined allocation concealment ${ }^{14,15}$ and undetermined initial group assembly. ${ }^{15}$ All were deemed to have high risk of bias due to confounding factors, poor randomisation and questionable blinding.

Reassuringly the conclusions (particularly relating to quality and bias) on the three papers reviewed are similar to that found by EFSA when it looked at the relationship between the consumption of xylitol lozenges and caries reduction. The EFSA reviewed three papers (two papers in common with this review) and concluded that these were weak due to lack of effect, poor randomisation and 'insufficient control for confounders'.

The authors stated that they did not have any commercial interest, but there was no discussion as to whether the review was funded externally. The three reviewed papers all had financial support through dental associations and xylitol producers but this financial support is unlikely to have altered the overall conclusions of the review.

\section{Brett G Duane}

Department of Public Health, NHS Fife, Cameron Hospital, Windygates, Fife, UK

1. Chambrone L, Chambrone D, Pustiglioni FE, Chambrone LA, Lima LA. Can subepithelia connective tissue grafts be considered the gold standard procedure for the treatment of Miller Class I and class II recession-type defects? I Dent 2008; 36: 659-671.

2. Scheinin A, Mäkinen KK, Ylitalo K. Turku sugar studies. V. Final report on the effect of sucrose, fructose and xylitol diets on the caries incidence in man. Acta Odontol Scand 1976; 34: 179-216.

3. Soderling E. Controversies around Xylitol. Eur / Dent 2009; 3: 81-82.

4. European Food Safety Authority. Xylitol chewing gum/pastilles and reduction of the risk of tooth decay. EFSA / 2008; 852: 1-15. Available at: http://www.efsa.europa.eu/ de/scdocs/doc/852.pdf) [accessed December $2^{\text {nd }} 2011$ ]

5. Vadeboncoeur C, Trahan L, Mouton C, Mayrand D. Effect of xylitol on the growth and glycolysis of acidogenic oral bacteria. / Dent Res 1983; 62: 882-884.

6. Loesche W], Grossman NS, Earnest R, Corpron R. The effect of chewing xylitol gum on the plaque and saliva levels of Streptococcus mutans. J Am Dent Assoc 1984; 108: 587-592.

7. Milgrom P, Ly KA, Roberts MC, Rothen M, Mueller G, Yamaguchi DK. Mutans streptococci dose response to xylitol chewing gum. J Dent Res 2006; 85: 177-181.

8. Campus G, Cagetti MG, Sacco G, Solinas G, Mastroberardino S, Lingstrom P. Six months of daily high-dose xylitol in high-risk schoolchildren: a randomized clinical trial on plaque $\mathrm{pH}$ and salivary mutans streptococci. Caries Res 2009; 43: 455-461.

9. Ly KA, Riedy CA, Milgrom P, Rothen M, Roberts MC, Zhou L. Xylitol gummy bear snacks: a school-based randomized clinical trial. BMC Oral Health 2008; 8: 20

10. Milgrom P, Ly KA, Tut OK, et al. Xylitol pediatric topical oral syrup to prevent dental caries: a double-blind randomized clinical trial of efficacy. Arch Pediatr Adolesc Med 2009; 163: 601-607.

11. van Loveren C, Aartman IH. The PICO (Patient Intervention Comparison Outcome) question. Ned Tijdschr Tandheelkd 2007; 114: 172-178.

12. Simes RJ. Publication bias: the case for an international registry of clinical trials. J Clin Oncol 1986; 4: 1529-1541.

13. Stecksen-Blicks C, Holgerson PL, Twetman S. Effect of xylitol and xylitol-fluoride lozenges on approximal caries development in high-caries risk-children. Int Paediatric Dent 2008; 18: 170-177.

14. Alanen P, Isokangas $P$, Gutmann K. Xylitol candies in caries prevention: results of a field study in Estonian children. Community Dent Oral Epidemiol 2001; 28: 218-224.

15. Honkala E, Honkala S, Shyama M, Al-Mutawa SA. Field trial on caries prevention with xylitol candies among disabled school students. Caries Res 2006; 40: 508-513

Evidence-Based Dentistry (2012) 13, 7-8. doi:10.1038/sj.ebd.6400836 